\title{
NOTES SUR L'ÉLEVAGE DES BLACK-BASS
}

\author{
par L. POMMIER \\ Trésorier de la Fédération Nationale de la Piscieulture
}

L'élevage des poissons est toujours délicat pendant la période d'alevinage. Il l'est particulièrement pour les poissons tels que : Truites, Brochets et Bass qui s'imposent par leurs qualités incontestables à plusieurs points de vue, mais ne se gênent pas, dans certaines circonstances, de s'attaquer aux plus faibles individus des autres espèces.

En ce qui concerne la Truite, les précautions dont il va être parlé n'ont pas d'intérêt, l'élevage se faisant artificiellement, en bacs constamment surveillés, où ne se trouve aucune autre espèce.

Il n'en est pas de même de l'alevinage en étangs du Brochet, ni du Black-Bass.

En ce qui concerne ce dernier, certaines remarques ont été faites et peuvent intéresser les éleveurs.

Pour l'alevinage du Black-Bass, les reproducteurs sont placés à raison d'une vingtaine de têtes par étangs de deux ou trois hectares.

Il est d'usage de placer dans les mêmes étangs des reproducteurs Carpes et Gardons.

Dans une Pisciculture bien suivie, la mise en présence de ces espèces n'a pas seulement pour résultat, en fin de saison, une belle production de jeunes Bass, mais, en même temps, une production particulièrement intéressante d'alevins de Carpes et même de Gardons.

Il est; en effet, facile de comprendre que, parmi les tout jeunes alevins de Carpes et de Gardons, ce sont les moins forts et les moins résistants qui sont les premiers à se trouver la proie des Bass. Les plus forts tiennent le coup et, à la pêche d'automne, ceux-ci, automatiquement sélectionnés et débarrassés des moins forts que l'étang n'a pas à nourrir, sont nettement plus beaux que dans un étang où il n'y a pas de Bass.

Mais, pour obtenir un tel résultat, il est une condition indispensable à réaliser. II faut que les petites Carpettes produites apparaissent dans l'étang en temps convenable, c'est-à-dire ḋ un moment où les alevins de Black-Bass n'ont pas pris assez de croissance pour s'attaquer trop facilement aux jeunes alevins de Carpes.

$\mathrm{Si}$, en effet, ils se trouvent en présence de jeunes poissons d'une dimension approximativement la même que la leur, ils seront amenés à ne détruire que les Carpettes les plus faibles et laisseront les plus fortes, qui se transformeront ensuite en alevins de très belle condition.

Au contraire, si l'alevinage des Bass se produit à une époque prématurée, 
c'est-à-dire bien avant que les Carpettes elles-mêmes apparaissent, celles-ci se trouveront trop petites et trop faibles pour se défendre des alevins de Bass. Ceux-ci, passé le moment de la période printanière d'abondance du zoo-plancton, seront déjà forts, de dimensions dépassant de beaucoup celles des Carpettes et seront donc tentés par cette population de tout premier âge à laquelle ils s'attaqueront trop facilement. Il en serait de meme des Gardons.

En conclusion, l'alevinage du Black-Bass, en présence de celui de la Carpe, bien conditionné, peut donner d'excellents résultats et permettre la production d'alevins de Carpes, particulièrement forts et beaux, mais à la condition indispensable de veiller à ce que la fraye des Black-Bass et celle des Carpes se produisent au cours des mêmes journées, ou à peu près.

Si l'empoissonnement en reproducteurs Carpes et Gardons est réalisé trop tard, et que la fraye de ces deux espèces se produise trop tardivement, après celle des Black-Bass, il y a bien des chances pour que les petites Carpes et les petits Gardons en souffrent sérieusement. 